\title{
Grid conected inverter for alternative sources of energy
}

\author{
Ryszard Porada ${ }^{1, *}$, Adam Gulczyński ${ }^{1}$ \\ ${ }^{1}$ Poznan University of Technology, 60-965 Poznań, Poland
}

\begin{abstract}
The general characteristic of power electronic couplers for alternative energy sources with electrical power grid was presented. The power electronic current source, working as a closed loop system, generating sine wave shaped current transfering the energy to the grid was discussed. The chosen results of simulation model of such system for sinusoidal reference signals were presented. The test results of the laboratory prototype were also presented.
\end{abstract}

\section{Introduction}

The energy obtained from alternative sources of energy may be used for supplying local, individual AC voltage receivers or transferred directly to the electrical power grid. For this purpose, the power electronic voltage inverters with voltage output or working as controllable current sources, are used. Controlling of such devices is a difficult task because of presence of nonlinearity effects [1,2].

In this paper the studies of power electronic coupler for low voltage alternative energy source (i.e. low voltage solar panel) with electrical power grid, which requires the matching transformer, were presented. The structure of the system and the method of generated current synchronization with grid voltage were shown. Selected results of simulation tests with a discrete corrector, as well as test results of a laboratory system test are included.

\section{Structure of the system}

The block diagram of the 1-phase power electronic current source cooperating with electrical power grid is presented on figure 1 .

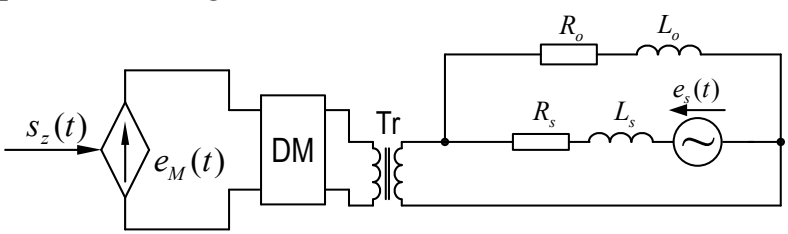

Fig. 1. The structure of the current source conected to grid.

The system consists of controllable modulated voltage source $e_{M}(t)$ as the voltage inverter with current output, supplied from the DC voltage source. The control signal $s_{z}(t)$ is generated by the corrector of the main track in closed current feedback loop system, defined according to the rules presented in the works $[3,4]$. On the output of modulated voltage source is passive demodulator (DM block), which acts like lowpass filter.
Two structures were adopted- inductie in series or $L C L$ filter type $T$. Becuase of voltage match the transfomer with properly chosen voltage ratio was used. The output of the transformer is connected to the electrical power grid, represented on the schematic by serial $e_{s}(t) R_{s} L_{s}$ branch. The other receivers are represented by serial $R_{o} L_{o}$ branch.

\section{Simulation tests}

The simulation test were conducted to initially select parameters of the corector due to laboratory model of one phase inveter, with two types of modulationunipolar and bipolar.

The following parameters of the system were adopted: supply voltage of the inverter $50[\mathrm{~V}]$, carrier frequency of PWM modulation $f_{P W M}=12,5 \mathrm{kHz}$. Parameters of the coupling choke: $R_{s p}=0,09[\Omega], L_{s p}=$ $1,1[\mathrm{mH}]$, LC filter: $R_{L}=0,05[\Omega], L_{L}=440[\mu \mathrm{H}], C=$ $44[\mu \mathrm{F}]$. The power grid is modeled by a serial branch with parameters $R_{s}=0,005[\Omega], L_{s}=55[\mu \mathrm{H}]$. The other receivers are represented by the serial branch with parameters $R_{o}=19,55[\Omega], L_{o}=385[\mu \mathrm{H}](\cos \varphi=0,85)$.
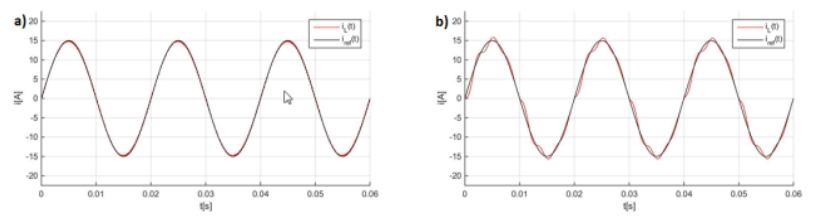

Fig. 2. Current and voltage waveforms of the system for current source: a) without LC filter; b) with LC filter.

Simulation studies for the discrete version of the corrector were performed in the Matlab / Simulink ${ }^{\circledR}$. The dead time for PWM modulation with the $t_{m}=2[\mu \mathrm{s}$ ] was also taken into account for the proper control of the inverter. The few selected results of studies of a unipolar modulation system (better frequency properties) and the shape of a sinusoidal current of $15 \mathrm{~A}$ are shown in fig.2. The corrector of the closed feedback loop system was chosen in manner to achieve the narrowband features of

Corresponding author: adam.gulczynski@put.poznan.pl 
the system, hence the lack of carrier components in the current waveforms. The presence of low-frequency components for the LCL filter is the result of the dead time.

\section{Laboratory prototype tests}

Experimental studies of the physical power source system in the 1-phase version were carried out on the basis of a converter with the IPM PM50RSA120 transistor module, controlled by the ALS-G3-1369 starter kit with Analog Devices ADSP-21369 SHARCTM floating-point signal processor. The voltage supply to the inverter is a laboratory power supply with a voltage of $50 \mathrm{~V}$ and a current capacity of $15 \mathrm{~A}$.

The control algorithm has been implemented in the microprocessor system. The block diagram of the control system is shown in figure 3 .

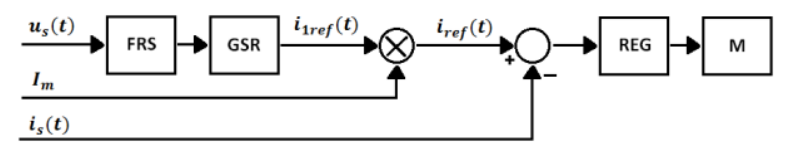

Fig. 3. Block diagram of the grid current control system.

Synchronization of the current of the transformer's secondary side (grid) with the mains voltage was performed using a moving averae as a low-pass filter. The voltage of the $u_{s}(t)$ was sampled, then filtered through the moving average filter and stored in a circular register (FRS block). The samples of a shifted in phase sine wave with respect to the grid voltage were obtained. The obtained samples were given on the peak value detector (GSR block). Next, the scaling of this waveform and the phase correction (compensation of phase characteristics of the average mobile filter) were made. Finally, a unit input signal for the current $i_{\text {ref }}(t)$ was obtained - figure 4.

The amplitude of the grid current is the result of the set power that should be transfered to the grid. The reference current is compared to the actual grid current, and then, as a control error, is fed by the regulator (corrector) to the modulator that generates switching signals for the inverter transistors.

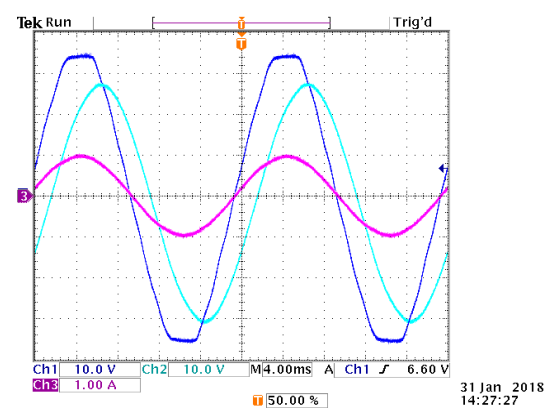

Fig. 4. Waveforms of: grid voltage (navy blue), filtered grid voltage (blue) and unit reference signal (purple).

The chosen study results of the laboratory prototype were presented on the figures 5 and 6 .

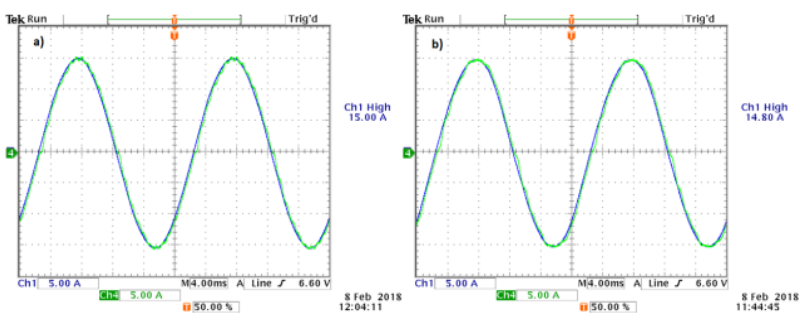

Fig. 5. Transformer's primary side current waveforms for the system a) without the LC filter ; b) with LC filter.

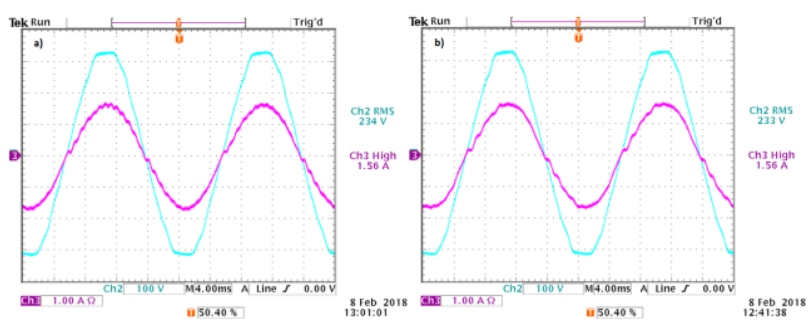

Fig. 6. Grid's voltage and current waveforms for the system: a) without the LC filter; b) with LC filter.

The tests were carried out for two versions of the passive demodulator - series inductance $\mathrm{L}$ and $\mathrm{T}$ type LCL and unipolar modulation. In the practical system, the value of deformation of the current transfered to the grid in both cases has a similar value $(<5 \%)$, which is an acceptable value in real life applications. A positive aspect of the obtained results is the low content of high frequency components in the current waveforms.

\section{Summary}

The results of laboratory tests indicate the need to perform some changes in the corrector system, controlling the physical system. The use of an LCL filter at the output of the inverter has little influence on the level of harmonic current distortions, however, it significantly changes the shape of the voltage of the transformer primary winding, which can affect the energy efficiency of the entire energy conversion path.

\section{References}

1. W Grega, Methods and algorithms of digital control in centralized and expanded systems (in polish), UWND AGH (Kraków, 2004)

2. N. Mohan, T.M. Undeland, W.P. Robbins, Power Electronics: Converters, Application and Design. JWS (New York, 2001)

3. R. Porada, Pozn. Univ. Technol. Acad. J. Electr. Eng. 87, 313-322 (2016)

4. R. Porada, A. Gulczyński, Pozn. Univ. Technol. Acad. J. Electr. Eng. 87, 225-233 (2016) 